\title{
Anomalous Raman spectra and thickness-dependent electronic properties of WSe $\mathrm{W}_{2}$
}

\author{
H. Sahin,,$^{1, *}$ S. Tongay, ${ }^{2, \dagger}$ S. Horzum, ${ }^{1,3}$ W. Fan, ${ }^{2}$ J. Zhou, ${ }^{2}$ J. Li,${ }^{4}$ J. Wu, ${ }^{2,5}$ and F. M. Peeters ${ }^{1}$ \\ ${ }^{1}$ Department of Physics, University of Antwerp, Groenenborgerlaan 171, B-2020 Antwerpen, Belgium \\ ${ }^{2}$ Department of Materials Science and Engineering, University of California, Berkeley, California 94720, USA \\ ${ }^{3}$ Department of Engineering Physics, Faculty of Engineering, Ankara University, 06100 Ankara, Turkey \\ ${ }^{4}$ Institute of Semiconductors, Chinese Academy of Sciences, P.O. Box 912, Beijing 100083, People's Republic of China \\ ${ }^{5}$ Materials Sciences Division, Lawrence Berkeley National Laboratory, Berkeley, California 94720, USA \\ (Received 13 February 2013; revised manuscript received 8 March 2013; published 5 April 2013)
}

\begin{abstract}
Typical Raman spectra of transition-metal dichalcogenides (TMDs) display two prominent peaks, $E_{2 g}$ and $A_{1 g}$, that are well separated from each other. We find that these modes are degenerate in bulk $\mathrm{WSe}_{2}$ yielding one single Raman peak in contrast to other TMDs. As the dimensionality is lowered, the observed peak splits in two. In contrast, our ab initio calculations predict that the degeneracy is retained even for $\mathrm{WSe}_{2}$ monolayers. Interestingly, for minuscule biaxial strain, the degeneracy is preserved, but once the crystal symmetry is broken by a small uniaxial strain, the degeneracy is lifted. Our calculated phonon dispersion for uniaxially strained $\mathrm{WSe}_{2}$ shows a good match to the measured Raman spectrum, which suggests that uniaxial strain exists in $\mathrm{WSe}_{2}$ flakes, possibly induced during the sample preparation and/or as a result of the interaction between $\mathrm{WSe}_{2}$ and the substrate. Furthermore, we find that $\mathrm{WSe}_{2}$ undergoes an indirect-to-direct band-gap transition from bulk to monolayers, which is ubiquitous for semiconducting TMDs. These results not only allow us to understand the vibrational and electronic properties of $\mathrm{WSe}_{2}$, but also point to effects of the interaction between the monolayer TMDs and the substrate on the vibrational and electronic properties.
\end{abstract}

DOI: 10.1103/PhysRevB.87.165409

PACS number(s): 63.22.Np, 66.30.Pa, 68.65.Pq, 81.16.Pr

\section{INTRODUCTION}

Owing to its extraordinary properties, ${ }^{1,2}$ graphene has already been implemented in various kinds of applications ${ }^{3,4}$ and has led to the emergence of a new class of materials: ultrathin two-dimensional crystal structures. Nowadays, among the members of this new era, the ultrathin transitionmetal dichalcogenides (TMDs) in particular have attracted considerable interest. ${ }^{5-8}$ Even though they are only a few atoms thick, the $M X_{2}$-type structures have remarkable chemical and mechanical stability $6,7,9$ and offer new physics through various quantum confinement effects amplified in quasi two dimensions. ${ }^{10-13}$ As a result of this confinement effect, the band gap increases and transforms to a direct band gap with a decreasing number of layers, which makes them promising candidates for nanoscale field-effect transistors and solar cell applications. ${ }^{14,15}$ Recently, the possibility of vacancy creation in TMDs under electron irradiation, ${ }^{16}$ band-gap transition in tungsten dichalcogenides, ${ }^{17}$ the existence of tightly bound negative trions,${ }^{18}$ and strain-engineered electronic properties have been reported. ${ }^{19}$ Furthermore, we reported that with strain application, $\mathrm{MoSe}_{2}$ and possibly other TMDs show a significant red shift in their Raman spectrum and undergo a direct-to-indirect band-gap transition. ${ }^{20}$

The synthesis and characterization of tungsten diselenide $\left(\mathrm{WSe}_{2}\right)$ has been an active field of research, with applications in photovoltaic and photoconductive devices, and recently monolayer $\mathrm{WSe}_{2}$ has become a popular choice for nanoscale devices. ${ }^{21}$ Here, we present an experimental and theoretical investigation of the electronic properties and lattice dynamics of bulk, few-layer, and single-layer $\mathrm{WSe}_{2}$. We find that the $A_{1 g}$ and $E_{2 g}$ modes are almost degenerate for bulk $\mathrm{WSe}_{2}$, whereas these modes are well separated for other members $\left(\mathrm{MoS}_{2}\right.$, $\mathrm{MoSe}_{2}$, and $\mathrm{WS}_{2}$ ) of the TMDs. Interestingly, this degeneracy is lifted as the dimensionality is lowered from three dimensions
(3D) (bulk limit) to 2D (monolayer), where the $A_{1 g}$ and $E_{2 g}$ modes are separated by $\sim 11 \mathrm{~cm}^{-1}$. The calculated vibrational spectrum shows that the lifting of the degeneracy is closely related to the uniaxial strain induced on monolayer $\mathrm{WSe}_{2}$ due to the interaction with the substrate and/or sample preparation procedure. Lastly, we show that the band gap of $\mathrm{WSe}_{2}$ goes through a rather "soft" indirect-to-direct band-gap crossover from bulk to monolayer and that the band gap shows almost triple-band degeneracy for bi- and trilayers, as evidenced by our photoluminescence measurements.

\section{EXPERIMENTAL DETAILS AND COMPUTATIONAL METHODOLOGY}

Monolayer and few-layer $\mathrm{WSe}_{2}$ flakes were exfoliated from $\mathrm{WSe}_{2}$ single crystals (2Dsemiconductors.com) onto $90 \mathrm{~nm}$ $\mathrm{SiO}_{2} / \mathrm{Si}$ (MTI Inc.) substrates using a conventional mechanical exfoliation technique. The thickness of the $\mathrm{WSe}_{2}$ flakes was confirmed by three complementary methods: atomic force microscopy (AFM), Raman spectroscopy, and photoluminescence (PL). Noncontact AFM line scans on the monolayer $\mathrm{WSe}_{2}$ typically resulted in $\sim 0.7 \mathrm{~nm}$ step height, corresponding to a single unit-cell lattice constant for $\mathrm{WSe}_{2}$ [see Fig. 1(d)]. PL and Raman measurements were performed using a very low power intensity $\left(10 \mu \mathrm{W} / \mu \mathrm{m}^{2}\right)$ on a $2-3 \mu \mathrm{m}^{2}$ circle to avoid local heating or damaging effects. The results presented in this paper were reproduced on more than 50 samples.

Theoretical calculations for equilibrium and strained structures were carried out in the framework of density functional theory (DFT), using the projector augmented wave (PAW) method $^{22}$ as implemented in the VASP code. ${ }^{23}$ The generalized gradient approximation (GGA) of Perdew-Burke-Ernzerhof (PBE) was used for the exchange-correlation functional. ${ }^{24} \mathrm{To}$ calculate the Raman spectrum of single-layer $\mathrm{WSe}_{2}$ under 
biaxial (uniaxial) strain, a hexagonal (rectangular) unit cell with one side parallel to the direction of stretch is taken into account. Phonon frequencies and phonon eigenvectors were calculated by using both the density functional perturbation theory (DFPT) ${ }^{25}$ and the small displacement method. ${ }^{26}$

\section{RESULTS AND DISCUSSIONS}

\section{A. Thickness-dependent electronic properties}

Similar to graphite, $\mathrm{WSe}_{2}$ crystals possess a lamellar structure with Bernal stacking, where the individual layers are
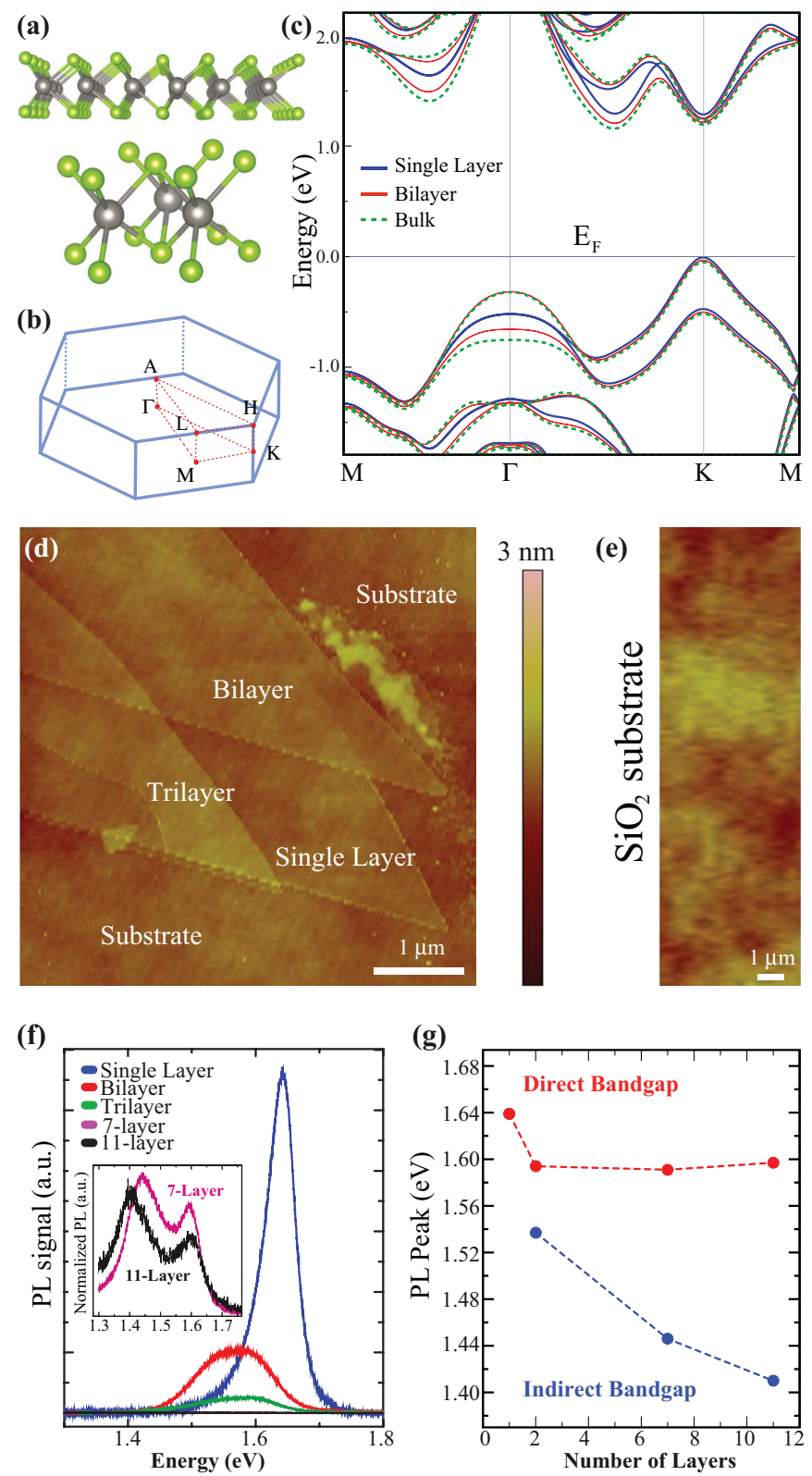

FIG. 1. (Color online) (a) Side and tilted view of single-layer $\mathrm{WSe}_{2}$. (b) Brillouin zone of $\mathrm{WSe}_{2}$ and (c) GGA + SO (spin-orbit) band structures. The Fermi level is set to zero. Blue, red, and green colors are for single-layer, bilayer, and bulk structures, respectively. Atomic force microscopy (AFM) images taken on (d) $\mathrm{WSe}_{2} / \mathrm{SiO}_{2}$ and (e) bare $\mathrm{SiO}_{2}$ substrate. (f) PL measurements on $\mathrm{WSe}_{2}$ flakes with various thicknesses and $(\mathrm{g})$ peak positions.
TABLE I. Calculated direct and indirect transitions between the VBM and CBM. The difference between the first and second CBM at the $K$ point is also given as $\Delta^{\mathrm{CBM}}(K)$. The experimental value is given in parentheses. All energies are given in $\mathrm{eV}$.

\begin{tabular}{lccccc}
\hline \hline WSe $_{2}$ & $\Gamma \rightarrow \Gamma-K$ & $\Gamma \rightarrow K$ & $K \rightarrow K$ & $K \rightarrow \Gamma-K$ & $\Delta^{C B M}(K)$ \\
\hline Bulk & 1.48 & 1.52 & 1.25 & 1.21 & 0.04 \\
Bilayer & 1.51 & 1.52 & 1.24 & 1.23 & 0.04 \\
Single layer & 1.83 & 1.78 & $1.25(1.64)$ & 1.30 & 0.04 \\
\hline \hline
\end{tabular}

weakly coupled to the adjacent layers by van der Waals (vdW) forces. As shown in Figs. 1(a) and 1(b), each monolayer $\mathrm{WSe}_{2}$ $\left(1 \mathrm{H}-\mathrm{WSe}_{2}\right)$ consists of $\mathrm{Se}-\mathrm{W}-\mathrm{Se}$ atoms wherein tungsten atoms are sandwiched between trigonally arranged sheets of selenium atoms. Our calculations reveal that bulk $\mathrm{WSe}_{2}$ has an $1.21 \mathrm{eV}$ indirect band gap, where the valence-band maximum (VBM) is located at the $K$ symmetry point while the conduction-band minimum $(\mathrm{CBM})$ is along the $\Gamma-K$ direction [Fig. 1(c), green dashed curve]. As shown in Table I conduction bands located at $K$ and $\Gamma-K$ are only separated by $0.04 \mathrm{eV}$ in energy and hence we expect that their electronic properties will be strained and dimensionality dependent as a result of significant changes in the hybridization. Since bulk $\mathrm{WSe}_{2}$ is an indirect band-gap semiconductor, the photoluminescence signal is expected to be rather weak for bulk $\mathrm{WSe}_{2}$, as observed in Fig. 1(f). Interestingly, 7 to 11 layers display two distinct PL peaks (hot luminescence) located at $1.39 \mathrm{eV}$ and $1.59 \mathrm{eV}$, where the former probes the indirect band gap $(\Gamma$ to $\Gamma-K)$ and the latter is associated with the direct band-gap transition ( $K$ to $K$ ) [see inset of Fig. 1(f)]. We note that the overall PL signal measured on few-layer flakes is orders of magnitude weaker in intensity as compared to bilayer and monolayer $\mathrm{WSe}_{2}$.

Electronic band structure calculations for bilayer and monolayer $\mathrm{WSe}_{2}$ show that the band gap increases from $1.21 \mathrm{eV}$ in bulk to $1.23 \mathrm{eV}$ (direct-indirect, almost degenerate) for bilayer and $1.25 \mathrm{eV}$ (direct) for monolayer $\mathrm{WSe}_{2}$ [see Fig. 1(c)]. The experimentally observed PL signal for bulk, bilayer, and monolayer $\mathrm{WSe}_{2}$ peaks at $1.57,1.57$, and $1.64 \mathrm{eV}$, respectively [Fig. 1(f)]. We see that in contrast to the general case, for $\mathrm{WSe}_{2}$, LDA, and GGA, band dispersions differ significantly. While GGA finds single-layer $\mathrm{WSe}_{2}$ as a semiconductor with a direct band gap of $1.56 \mathrm{eV}$, LDA gives an indirect band gap of $1.68 \mathrm{eV}$. Here, both LDA and GGA finds the VBM at the $K$ symmetry point and the CBM is located at the $K$ (in between $\Gamma$ and $K$ ) point in GGA (LDA). It appears that the GGA exchange correlation better approximates the ground-state characteristics of the $\mathrm{WSe}_{2}$ crystal structure.

Here it is also worth noting that the spin-orbit interaction results in two significant effects on the electronic band dispersion: (i) shifting down the valence-band energies at the $\Gamma$ point, and (ii) band splitting at the vicinity of the $K$ and $M$ symmetry points. Clearly, the existence of an intrinsic electric field breaks the inversion symmetry in the crystal structure. Therefore, it is seen that the degeneracy of the doubly degenerate valence and conduction bands of single-layer $\mathrm{WSe}_{2}$ is removed by spin-orbit interaction, and a band splitting occurs. Furthermore, when the spin-orbit interaction is taken into account in our calculations, conduction-band edges at the $K$ and $K-\Gamma$ points show band splitting that allows various direct 
and indirect transitions (even in few-layered $\mathrm{WSe}_{2}$ structures). In our study, all of the band structure calculations presented are performed by considering spin-orbit interaction together with GGA.

Previously, the indirect-to-direct band crossover has been observed in other transition-metal dichalcogenides ${ }^{10,14,20}$ and is consistent with our results. However, we note that the indirect-to-direct transition is rather steep for $\mathrm{MoS}_{2}$ where its bilayer form is an indirect band-gap $(1.6 \mathrm{eV})$ semiconductor, while the monolayer structure has a direct band gap $(1.9 \mathrm{eV})$. In contrast, bilayer $\mathrm{WSe}_{2}$ possesses almost triple-band-gap degeneracy where the $K \rightarrow K$ and $K \rightarrow \Gamma-K$ gap values are almost degenerate and the difference between first and second CBMs is $\triangle^{C B M}(K)=40 \mathrm{meV}$, which is close to thermal broadening $(\sim 30 \mathrm{meV})$. As a result of this band degeneracy, the integrated PL intensity [Fig. 1(f)] of bilayer $\mathrm{WSe}_{2}$ is of the same order of magnitude as in monolayers, i.e., $I_{1 L} / I_{2 L} \sim 1-10$, which compares with 100-1000 for $\mathrm{MoS}_{2}$. We also note that the PL signal for bilayer $\mathrm{WSe}_{2}$ is rather broad and can be described by at least two Lorentzian peaks. As the number of layers increases, the band degeneracy is gradually lifted, $\mathrm{WSe}_{2}$ becomes truly an indirect band-gap semiconductor, and the PL intensity decreases by orders of magnitude.

\section{B. Anomalous lattice vibrations: Breaking the degeneracy of $A_{1 g}$ and $E_{2 g}$ modes at reduced dimensions}

Next we turn our attention to the anomalous lattice vibrations of $\mathrm{WSe}_{2}$. For the determination of lattice dynamics, we use Raman spectroscopy which is one of the most useful nondestructive techniques for the characterization of low-dimensional materials. Our measurements reveal that the most prominent Raman peak for bulk $\mathrm{WSe}_{2}$ is located at $252.2 \mathrm{~cm}^{-1}$ [Fig. 2(a)], while other semiconducting TMDs are characterized by well-separated $E_{g}$ and $A_{g}$ Raman peaks. The stark difference between $\mathrm{WSe}_{2}$ and other TMDs already points towards an anomaly in the phonon dispersion of $\mathrm{WSe}_{2}$. Following from Fig. 2(b), calculated phonon dispersion confirms that bulk WSe ${ }_{2}$ displays only one strong vibrational mode around $250 \mathrm{~cm}^{-1}$, which is consistent with our experiments. Interestingly, for flakes thinner than four layers, an additional peak appears at roughly $5-11 \mathrm{~cm}^{-1}$ above the first-order Raman peak. We find that the frequency difference between these two peaks is 5,6 , and $11 \mathrm{~cm}^{-1}$ for trilayer, bilayer, and monolayer $\mathrm{WSe}_{2}$ flakes, respectively. This peak is absent in few-layer flakes and does not correspond to any new/additional Raman modes in the calculated phonon-dispersion curve, which describes the Raman spectrum well.

Bulk and single-layer $\mathrm{WSe}_{2}$ can be classified in the space groups $P 63 / m m c$ and $P 6 m 2$, respectively. Similar to the $\mathrm{MoS}_{2}$ and $\mathrm{MoSe}_{2}$ counterparts, ${ }^{20}$ the unit cell of bulk $\mathrm{WSe}_{2}$ has 18 phonon branches corresponding to 6 acoustic and 12 optical phonon modes. While the modes at $\sim 170(\sim 300) \mathrm{cm}^{-1}$ are fourfold (twofold) degenerate, six modes become almost degenerate at $\sim 250 \mathrm{~cm}^{-1}$ at the $\Gamma$ point. For a better understanding of the phonon spectrum and the prominent peak in the measurement, we present phonon symmetry representations of related modes and corresponding atomic motions in Figs. 2(b) and 2(c). It is seen that the fourfold branch at $\sim 170 \mathrm{~cm}^{-1}$ is
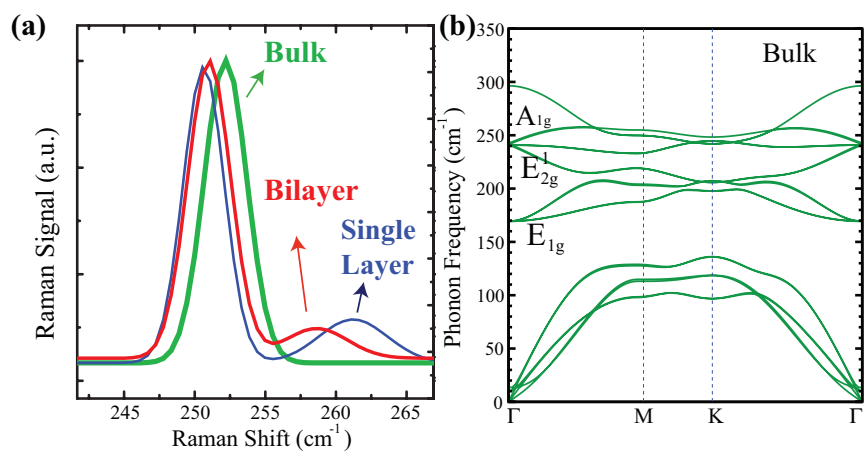

(c)

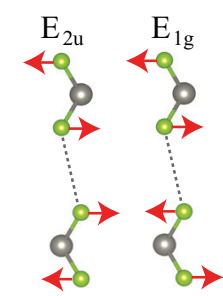

(d)
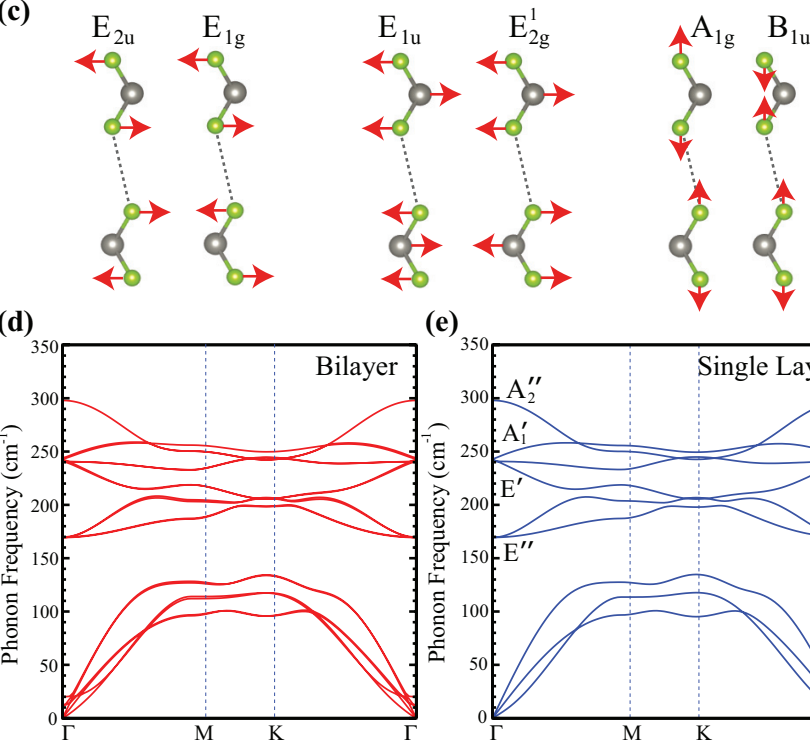

(e)

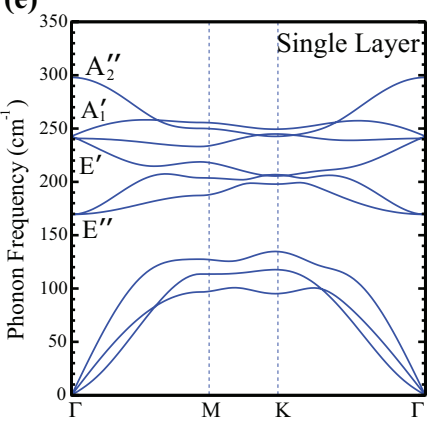

(f)
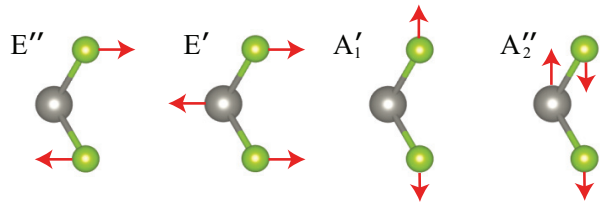

FIG. 2. (Color online) (a) Raman shift for single-layer, bilayer, and bulk $\mathrm{WSe}_{2}$. (b) Phonon spectrum of bulk $\mathrm{WSe}_{2}$ and (c) atomic displacements for optical modes between 100-250 $\mathrm{cm}^{-1}$. Phonon dispersions for (d) bilayer and (e) single-layer structure and (f) atomic displacements for optical phonon modes of single-layer $\mathrm{WSe}_{2}$.

formed by the vibrational motions corresponding to the $E_{2 u}$ and $E_{1 g}$ modes. Among these, only the $E_{1 g}$ mode is Raman active. However, in backscattering experiments on a surface perpendicular to the $c$ axis, the $E_{1 g}$ mode is forbidden and is not observed in our experiments. Furthermore, the decomposition of the sixfold phonon branch at $\sim 250 \mathrm{~cm}^{-1}$ (at the $\Gamma$ point) can be described as $\Gamma=2 E_{1 u}+2 E_{2 g}^{1}+B_{1 u}+A_{1 g}$. Among these, the $E_{2 g}$ and $A_{1 g}$ modes are Raman active and are degenerate in energy. As a result of this degeneracy, only one Raman peak can be observed in our measurements at $250 \mathrm{~cm}^{-1}$.

Next, we pay attention to the emergence of the second Raman peak in few- and single-layer $\mathrm{WSe}_{2}$ that appears at $\sim 11 \mathrm{~cm}^{-1}$ above the main peak. Although from bulk to singlelayer $\mathrm{WSe}_{2}$ there is no visible change in the calculated phonon dispersion [Figs. 2(b), 2(d), and 2(e)], our measurements show that a new peak develops [Fig. 2(a)] and this new peak appears 
(a)
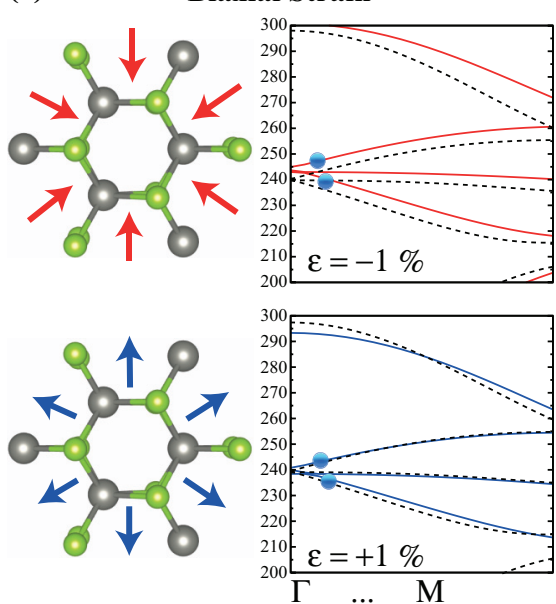

(b)
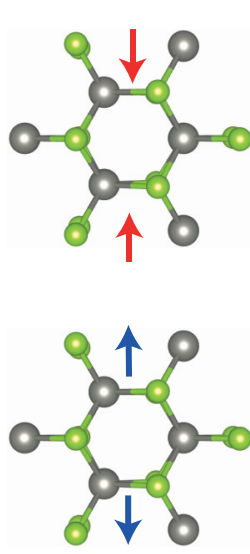

Uniaxial Strain (armchair)
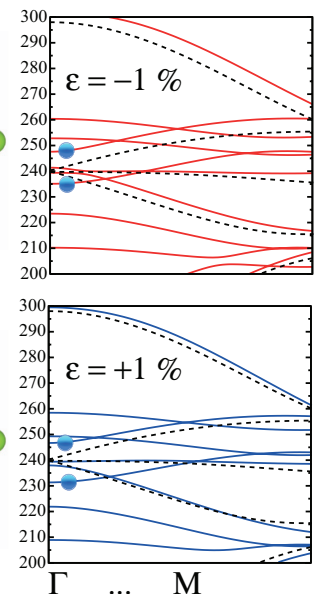
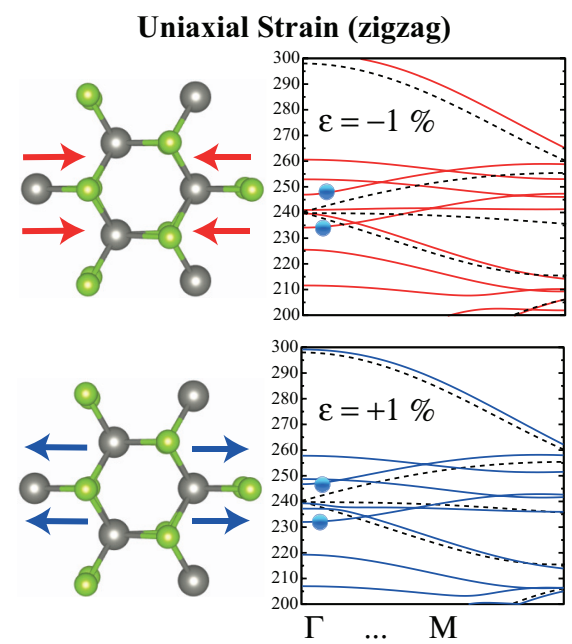

FIG. 3. (Color online) Application of biaxial, uniaxial-armchair, and uniaxial-zigzag strain to monolayer $\mathrm{WSe}_{2}$. Compressive and tensile strain are shown by inward and outward arrows, respectively. Phonon dispersion of unstrained $\mathrm{WSe}_{2}$ is shown by dashed line. Raman-active branches are labeled by blue dots.

to be very sensitive to the number of layers. For single-layer $\mathrm{WSe}_{2}$, decomposition of the vibration representation is calculated to be $\Gamma=2 E^{\prime \prime}+2 E^{\prime}+A_{1}^{\prime}+A_{2}^{\prime \prime}$. Here, the $E^{\prime \prime}, E^{\prime}$, and $A_{1}^{\prime}$ modes correspond to the $E_{1 g}, E_{2 g}$, and $A_{1 g}$ modes for bulk, respectively. Since the $E^{\prime}$ and $A_{1}$ modes are almost degenerate, only one Raman peak is expected to be observed in the experiment, which contradicts our experimental results. Before discussing the discrepancy more, we note that the presence of contaminants at the WSe2/interface and/or directly bonded to the TMD monolayer might cause small alterations to the Raman spectrum. However, if the contaminants are directly bonded (chemisorption) to the TMD, one would expect to observe drastic differences in the Raman spectrum as a result of renormalization of phonon dispersion. Since the overall monolayer Raman spectrum is similar to few layer, we eliminate this possibility. If the contaminants are chemically interacting locally, this would change the Raman signal from those local regions (Bloch waves intermix), but would be small compared to the overall Raman signal due to the geometrical considerations and reduced Raman sensitivity of the locally interacting region. To explain the emergence of the new peak, we consider external factors, such as compressive and tensile strain acting on few-layer and monolayer $\mathrm{WSe}_{2}$ flakes, which is likely to be induced by the interaction with the substrate.

To test the possibility of strain effects on $\mathrm{WSe}_{2}$ as the origin for such splitting of the Raman peak, we calculate the phonon dispersion of the monolayer $\mathrm{WSe}_{2}$ after applying $1 \%$ biaxial compressive and tensile strain. As seen from Fig. 3(a), such biaxial deformation only results in a collective softening/hardening of the vibrational modes and does not lift the degeneracy of the $E_{2 g}$ and $A_{1 g}$ modes ( $E^{\prime}$ and $A_{1}^{\prime}$ in single layer), implying that these modes remain degenerate as long as the hexagonal symmetry of the monolayer $\mathrm{WSe}_{2}$ is retained.

Next, we apply uniaxial strain that is likely to occur along with the biaxial strain on monolayer $\mathrm{WSe}_{2}$ exfoliated on an $\mathrm{Si} / \mathrm{SiO}_{2}$ surface. For hexagonally ordered crystal structures, uniaxial strain can be applied in two main directions: armchair and zigzag [Fig. 3(b)]. Interestingly, in the presence of uniaxial strain, the degeneracy of the branches forming the most prominent Raman peak is broken. While most of the modes between $200-280 \mathrm{~cm}^{-1}$ lose their symmetry that determines the Raman activity, our symmetry analysis shows that two of the branches that correspond to the $E^{\prime}$ (lower) and $A_{1}^{\prime}$ (upper) modes are still Raman active. For compressive (tensile) uniaxial strain along the armchair direction, the difference between the $E^{\prime}$ and $A_{1}^{\prime}$ modes is $16(18) \mathrm{cm}^{-1}$. Similarly, for compressive (tensile) strain along the zigzag direction, splitting is calculated to be $13(17) \mathrm{cm}^{-1}$. Here the $E^{\prime}-A_{1}^{\prime}$ splitting induced by uniaxial strain is in good agreement with our experimental data. It is worth noting that independent from the direction of both tensile and compressive strains, both have the same splitting effect on the Raman peaks.

\section{DISCUSSIONS AND CONCLUSIONS}

According to our phonon-dispersion calculations performed on bulk $\mathrm{WSe}_{2}$, the out-of-plane $\left(A_{1 g}\right)$ and in-plane $\left(E_{2 g}\right)$ modes are degenerate in energy, which is consistent with the experimental measurements taken on bulk $\mathrm{WSe}_{2}$. Experimentally, as the dimension is lowered from $3 \mathrm{D}$ to $2 \mathrm{D}$ (bulk to monolayers), the Raman mode located at $\sim 250 \mathrm{~cm}^{-1}$ splits in two peaks at $\sim 250 \mathrm{~cm}^{-1}$ and $\sim 261 \mathrm{~cm}^{-1}$. To provide an explanation for the broken degeneracy, we recalculate the phonon dispersion of monolayer $\mathrm{WSe}_{2}$ under uniaxial and biaxial strain/stress. Our theoretical results imply that the degeneracy of these two modes is lifted only if the crystal symmetry is broken, i.e., in the presence of uniaxial strain. When the monolayer $\mathrm{WSe}_{2}$ is under very little uniaxial strain or stress, our theoretical calculations show a good match with the experimental data. Considering the above arguments, we next argue about the possible origin of uniaxial strain on thin $\mathrm{WSe}_{2}$ flakes. Since our $\mathrm{SiO}_{2}$ substrates typically display 4-8 $\AA$ surface roughness [Figs. 1(d) and 1(e)] that is of the same order of a single unit-cell thickness, even perfect $\mathrm{SiO}_{2} / \mathrm{WSe}_{2}$ interface is likely to induce a mild strain to the $\mathrm{WSe}_{2}$ monolayer. The presence of the surface roughness on $\mathrm{SiO}_{2}$ 
can be observed clearly when compared to sapphire, which yields only 1-2 A surface roughness. Typically, these rough features are asymmetric in shape and therefore strain induced on the few-layer $\mathrm{WSe}_{2}$ is expected to have both a biaxial and uniaxial component. However, since the biaxial strain does not lift the degeneracy, only the uniaxial component of the total strain results in Raman peak splitting. Moreover, in the presence of residual contaminants at the $\mathrm{WSe}_{2} / \mathrm{SiO}_{2}$ interface and/or residues deposited after the exfoliation step, the effect of strain is likely to be amplified. From this perspective, monolayers are most affected by the strain effects, while thick flakes remain mostly unaltered. Another scenario might be associated with the unintentional strain induced during the exfoliation process. In such case, the $\mathrm{WSe}_{2}$ monolayers are deposited on the $\mathrm{SiO}_{2}$ substrates during the exfoliation and it is not necessarily related to the surface residue. However, we note that our results were confirmed on 50 different $\mathrm{WSe}_{2}$ monolayers where the same results have been found, supporting this scenario. Another indirect confirmation of the presence of uniaxial strain comes from the changes in the PL peak position. We also note that the band gap at the $K-K$ symmetry point is expected to be independent of the dimensionality. On the contrary, PL measurements show that the $K-K$ gap increases abruptly for the monolayer, likely due to the presence of small uniaxial strain [Fig. 1(g)], as is confirmed by our DFT calculations. We also point out that in the presence of a large density of defects, the degeneracy can be lifted. However, similar measurements taken on more than 50 independently prepared samples makes this case unlikely.

Lastly, we point out that the Raman spectrum of graphitic materials shows a strong sensitivity to the number of layers, which influences their electronic structure. A similar splitting effect in graphene's $G$ peak, which is due to the doubly degenerate zone-center $E_{2 g}$ mode, can be observed by modifying the electron-phonon interaction upon inhomogeneous doping. ${ }^{27,28}$ It was also shown that another mode splitting of the 2D peak of graphitic materials which is not seen in the first-order Raman spectra of defect-free samples can be driven by changes in the dimensionality. ${ }^{29}$ The essential difference from these is that here the mode splitting in the Raman spectrum of $\mathrm{WSe}_{2}$ originates from broken crystal symmetry.

In conclusion, phonon dispersion and the electronic properties of bulk to monolayer $\mathrm{WSe}_{2}$ have been studied both experimentally and theoretically. Unlike the other members of the TMDs, the $E_{2 g}$ and $A_{1 g}$ modes are degenerate in bulk $\mathrm{WSe}_{2}$ and the degeneracy is lifted as the dimension is lowered. Contrary to our experimental results, calculated phonon dispersion shows that these modes remain degenerate independent from the dimensionality. However, theoretically the degeneracy is only lifted when the crystal symmetry is broken, i.e., in the presence of uniaxial strain, which might be induced by the interaction with the substrate, residues, and/or exfoliation process. These results provide a deeper understanding of the vibrational properties of TMDs, especially on a material with unique phonon dispersion.

\section{ACKNOWLEDGMENTS}

This work was supported by the Flemish Science Foundation (FWO-Vl) and the Methusalem programme of the Flemish government. Computational resources were partially provided by TUBITAK ULAKBIM, High Performance and Grid Computing Center (TR-Grid e-Infrastructure). H.S. is supported by the FWO Pegasus Marie Curie Long Fellowship program. *hasan.sahin@ua.ac.be

†tongay@berkeley.edu

${ }^{1}$ K. S. Novoselov, A. K. Geim, S. V. Morozov, D. Jiang, S. C. Dubonos, I. V. Grigorieva, and A. A. Firsov, Science 306, 666 (2004).

${ }^{2}$ A. K. Geim and K. S. Novoselov, Nature Mater. 6, 183 (2007).

${ }^{3}$ X. Miao, S. Tongay, M. K. Petterson, K. Berke, A. G. Rinzler, B. R. Appleton, and A. F. Hebard, Nano Lett. 12, 2745 (2012).

${ }^{4}$ F. Schwierz, Nature Nanotechnol. 5, 487 (2010).

${ }^{5}$ R. A. Gordon, D. Yang, E. D. Crozier, D. T. Jiang, and R. F. Frindt, Phys. Rev. B 65, 125407 (2002).

${ }^{6}$ J. N. Coleman et al., Science 331, 568 (2011).

${ }^{7}$ Q. H. Wang, K. Kalantar-Zadeh, A. Kis, J. N. Coleman, and M. S. Strano, Nature Nanotechnol. 7, 699 (2012).

${ }^{8}$ S. Tongay, S. S. Varnoosfaderani, B. R. Appleton, J. Wu, and A. F. Hebard, Appl. Phys. Lett. 101, 123105 (2012).

${ }^{9}$ C. Ataca, H. Sahin, and S. Ciraci, J. Phys. Chem. C 116, 8983 (2012).

${ }^{10}$ K. F. Mak, C. Lee, J. Hone, J. Shan, and T. F. Heinz, Phys. Rev. Lett. 105, 136805 (2010).

${ }^{11}$ H. Peelaers and C. G. Van de Walle, Phys. Rev. B 86, 241401(R) (2012).
${ }^{12}$ S. W. Han, H. Kwon, S. K. Kim, S. Ryu, W. S. Yun, D. H. Kim, J. H. Hwang, J.-S. Kang, J. Baik, H. J. Shin, and S. C. Hong, Phys. Rev. B 84, 045409 (2011).

${ }^{13}$ J. K. Ellis, M. J. Lucero, and G. E. Scuseria, Appl. Phys. Lett. 99, 261908 (2011)

${ }^{14}$ S. Tongay, J. Zhou, C. Ataca, K. Lo, T. S. Matthews, J. Li, J. C. Grossman, and J. Wu, Nano Lett. 12, 5576 (2012).

${ }^{15}$ J. Kang, S. Tongay, J. Zhou, J. Li, and J. Wu, Appl. Phys. Lett. 102, 012111 (2013).

${ }^{16}$ H. P. Komsa, J. Kotakoski, S. Kurasch, O. Lehtinen, U. Kaiser, and

A. V. Krasheninnikov, Phys. Rev. Lett. 109, 035503 (2012).

${ }^{17}$ W. Zhao, Z. Ghorannevis, L. Chu, M. Toh, C. Kloc, P.-H. Tan, and G. Eda, ACS Nano 7, 791 (2013).

${ }^{18}$ K. F. Mak, K. He, C. Lee, G. H. Lee, J. Hone, T. F. Heinz, and J. Shan, Nature Mater. 12, 207 (2013).

${ }^{19}$ J. Feng, X. Qian, C. Huang, and J. Li, Nature Photon. 6, 866 (2012).

${ }^{20}$ S. Horzum, H. Sahin, S. Cahangirov, P. Cudazzo, A. Rubio, T. Serin, and F. M. Peeters, Phys. Rev. B 87, 125415 (2013).

${ }^{21}$ H. Fang, S. Chuang, T. C. Chang, K. Takei, T. Takahashi, and A. Javey, Nano Lett. 12, 3788 (2012).

${ }^{22}$ P. E. Blochl, Phys. Rev. B 50, 17953 (1994).

${ }^{23}$ G. Kresse and J. Hafner, Phys. Rev. B 47, 558 (1993).

${ }^{24}$ J. P. Perdew, K. Burke, and M. Ernzerhof, Phys. Rev. Lett. 77, 3865 (1996). 
${ }^{25}$ S. Baroni, S. de Gironcoli, A. Dal Corso, and P. Giannozzi, Rev. Mod. Phys. 73, 515 (2001).

${ }^{26}$ D. Alfe, Comput. Phys. Commun. 180, 2622 (2009).

${ }^{27}$ A. Gruneis, C. Attaccalite, A. Rubio, D. V. Vyalikh, S. L. Molodtsov, J. Fink, R. Follath, W. Eberhardt, B. Büchner, and T. Pichler, Phys. Rev. B 79, 205106 (2009).
${ }^{28}$ C. A. Howard, M. P. M. Dean, and F. Withers, Phys. Rev. B 84, 241404 (2011).

${ }^{29}$ A. C. Ferrari, J. C. Meyer, V. Scardaci, C. Casiraghi, M. Lazzeri, F. Mauri, S. Piscanec, D. Jiang, K. S. Novoselov, S. Roth, and A. K. Geim, Phys. Rev. Lett. 97, 187401 (2006). 